\title{
Stromal micropapillary component as a novel unfavorable prognostic factor of lung adenocarcinoma
}

\author{
Miki Ohe ${ }^{1,2,5}$, Tomoyuki Yokose ${ }^{1 *}$, Yuji Sakuma ${ }^{3}$, Yohei Miyagi ${ }^{3}$, Naoyuki Okamoto ${ }^{4}$, Sachie Osanai ${ }^{1}$, \\ Chikako Hasegawa ${ }^{1}$, Haruhiko Nakayama ${ }^{2}$, Yoichi Kameda', Kouzo Yamada ${ }^{2}$ and Takeshi Isobe ${ }^{5}$
}

\begin{abstract}
Background: Pulmonary adenocarcinomas with a micropapillary component having small papillary tufts and lacking a central fibrovascular core are thought to result in poor prognosis. However, the component consists of tumor cells often floating within alveolar spaces (aerogenous micropapillary component [AMPC]) rather than invading fibrotic stroma observed in other organs like breast (stromal invasive micropapillary component [SMPC]). We previously observed cases of lung adenocarcinoma with predominant SMPC that was associated with micropapillary growth of tumors in fibrotic stroma observed in other organs. We evaluated the incidence and clinicopathological characteristics of SMPC in lung adenocarcinoma cases.

Patients and Methods: We investigated the clinicopathological characteristics and prognostic significance of SMPC in lung adenocarcinoma cases by reviewing 559 patients who had undergone surgical resection. We examined the SMPC by performing immunohistochemical analysis with 17 antibodies and by genetic analysis with epidermal growth factor receptor (EGFR) and KRAS mutations.
\end{abstract}

Results: SMPC-positive (SMPC(+)) tumors were observed in 19 cases (3.4\%). The presence of SMPC was significantly associated with tumor size, advanced-stage disease, lymph node metastasis, pleural invasion, lymphatic invasion, and vascular invasion. Patients with SMPC(+) tumors had significantly poorer outcomes than those with SMPCnegative tumors. Multivariate analysis revealed that SMPC was a significant independent prognostic factor of lung adenocarcinoma, especially for disease-free survival of pathological stage I patients ( $p=0.035$ ). SMPC showed significantly higher expression of E-cadherin and lower expression of CD44 than the corresponding expression levels shown by AMPC and showed lower surfactant apoprotein A and phospho-c-Met expression level than corresponding expression levels shown by tumor cell components without a micropapillary component. Fourteen cases with SMPC(+) tumors (74\%) showed EGFR mutations, and none of them showed KRAS mutations.

Conclusions: SMPC(+) tumors are rare, but they may be associated with a poor prognosis and have different phenotypic and genotypic characteristics from those of AMPC(+) tumors.

Virtual Slides: The virtual slide(s) for this article can be found here: http://www.diagnosticpathology.diagnomx.eu/ vs/9433341526290040.

Keywords: lung adenocarcinoma, micropapillary component, stromal micropapillary component, aerogenous micropapillary component, prognostic factor

\footnotetext{
* Correspondence: yokose-t@kcch.jp

'Department of Pathology, Kanagawa Cancer Center, 1-1-2 Nakao, Asahi-ku,

Yokohama, Kanagawa, 241-0815, Japan

Full list of author information is available at the end of the article
} 


\section{Background}

A new lung adenocarcinoma classification system has been proposed by the International Association for the Study of Lung Cancer, American Thoracic Society, and European Respiratory Society (IASLC/ATS/ERS) [1]. In this classification, the micropapillary component (MPC) was recommended as a new subtype of lung adenocarcinoma in addition to the lepidic, acinar, papillary, and solid subtypes defined in the 2004 World Health Organization (WHO) classification [2]. MPC was defined as tumor cells growing in papillary tufts lacking fibrovascular cores and may float within alveolar spaces. MPC-predominant lung adenocarcinoma shows a high incidence of nodal metastasis and a poor prognosis [3-8]. MPCpredominant carcinomas developing in various other organs, such as the breast and urinary bladder, known as invasive micropapillary carcinoma, also have a poor prognosis. However, localization of MPC in the lungs is significantly different from that in the other organs; MPC in lung adenocarcinoma is distinguished by floating tumor cells within alveolar spaces (aerogenous micropapillary component, AMPC), while MPC in other organs has been observed primarily in the stroma as invasive components (stromal invasive micropapillary component, SMPC) [3,4].

Few studies have examined lung adenocarcinoma with SMPC $[9,10]$. Recently, we reported 2 cases of SMPCpredominant lung adenocarcinoma [9]. The proportion of SMPC in both tumors was greater than $50 \%$ in area. We observed that SMPC had a strong association with vascular invasion, similar to the cases of SMPC-predominant carcinoma in other organs. However, a largescale investigation on pulmonary SMPC has not been conducted.

The aims of this study included: (1) clarifying the incidence of SMPC in lung adenocarcinoma; (2) elucidating the clinicopathological characteristics of the tumor; and (3) determining the prognoses of the SMPC-positive $(\mathrm{SMPC}(+))$ tumors and comparing them with those of SMPC-negative (SMPC(-)) tumors. We reviewed 559 resected lung adenocarcinomas for this study with performing immunohistochemical and genetic analysis.

\section{Methods}

\section{Patients}

We analyzed 565 consecutive cases of primary lung adenocarcinoma treated by surgical resection at the Kanagawa Cancer Center between February 2007 and December 2010. Formalin fixation of the resected lung tissue was performed within 48 hours to reduce the loss of immunohistochemical antigen expression and degeneration of DNA. Six patients who had received preoperative chemotherapy were excluded. A total of 559 cases were enrolled in the study. The median follow-up time was 634.5 days (range, 28-1512 days). All patients provided informed consent, and the studies were performed according to the requirements of the institutional review board of Kanagawa Cancer Center.

\section{Pathological review}

Excised specimens were fixed in a solution of $10 \%$ buffered formaldehyde, and the sections were embedded in paraffin. Next, 4- $\mu$ m-thick sections, including the largest cut surface of the tumor, were prepared and stained using hematoxylin and eosin (HE) as well as alcian blue and elastica-van-Gieson (AB-EVG) to detect cytoplasmic mucin production and the elastic fiber framework. Lymphatic invasion and pulmonary metastasis were evaluated on HE sections. Vascular and pleural invasion was evaluated in AB-EVG sections. Sections were reviewed by 2 observers (M.O. and T.Y.) who were unaware of the clinical data. Tumor size was measured as the maximal diameter on the cut sections of the lung. Pathological stage was determined based on the criteria of the $7^{\text {th }}$ TNM classification of Union of International Cancer Control [11].

\section{Histological definition of micropapillary components}

Histopathological diagnosis of lung adenocarcinoma was determined according to the IASLC/ATS/ERS international multidisciplinary classification of lung adenocarcinoma [1]. Comprehensive histological subtyping was performed on the primary tumor and divided by percentage into 5 distinctive subtypes: lepidic, acinar, papillary, micropapillary, and solid, totaling $100 \%$ per tumor. We defined the subtype as positive when it occupied at least $1 \%$ of the entire tumor. We classified a micropapillary subtype into 2 components, AMPC and SMPC, using the following criteria: AMPC is widely recognized in the lungs as tumor cells floating within alveolar spaces, and SMPC includes papillary components consisting of tufts lacking central fibrovascular cores, surrounded by lacunar spaces and identified as invasive components in the stroma as previously described [9] (Figure 1A and 1B). Additionally, a tumor area without micropapillary components was defined as a non-micropapillary component (nMPC).

\section{Tumor tissue microarray (TMA) synthesis}

TMAs were constructed using a manual tissue-arraying instrument (KIN-4; Azumaya, Tokyo, Japan) as previously described [12], and specimens were punched using a stylet $3 \mathrm{~mm}$ in diameter.

\section{Immunohistochemistry}

The 17 antibodies used for immunohistochemical characterization of tumor cells in TMA in this study are listed in Table 1. Immunohistochemical staining was 


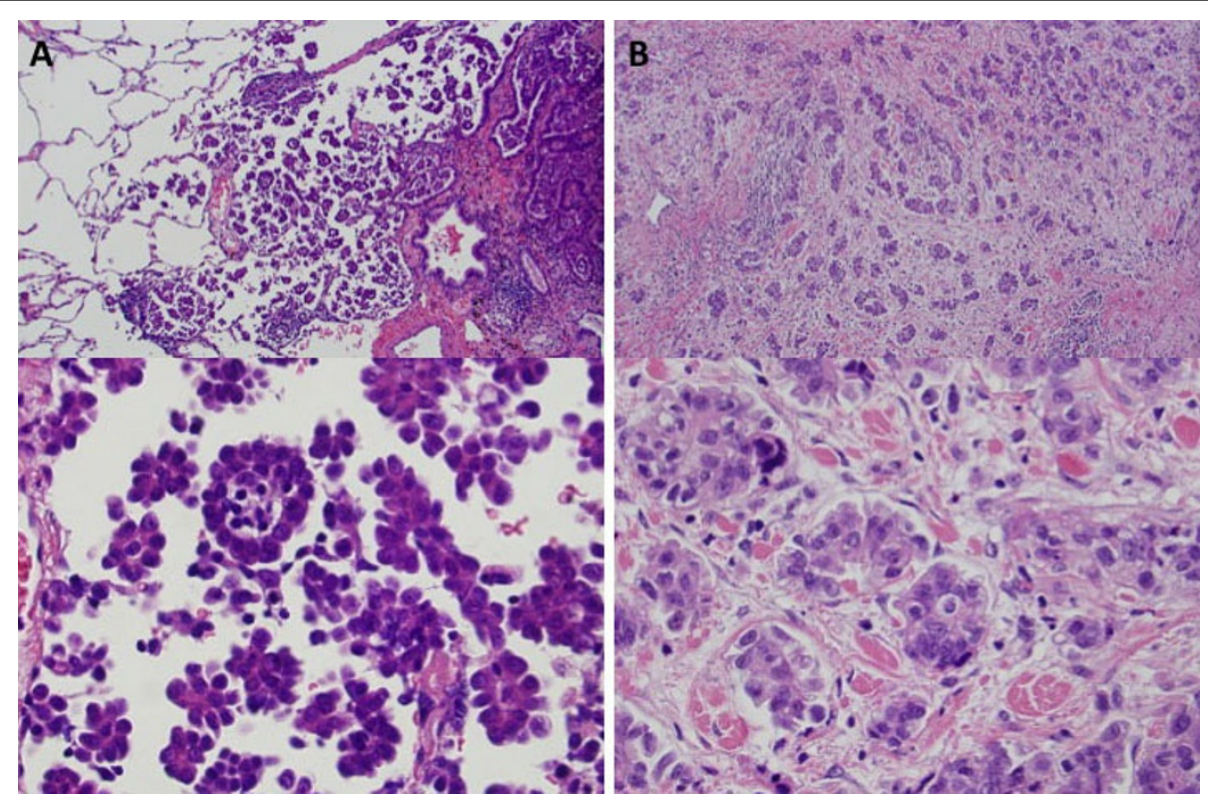

Figure 1 Microscopic features of micropapillary component in the lung adenocarcinoma stained with hematoxylin-eosin (HE). A, AMPC. AMPC is the micropapillary component in which tumor cells are floating within alveolar spaces. B, SMPC. SMPC are tumor cells observed in the stroma and consisting of a papillary component with a tuft lacking central fibrovascular cores surrounded by lacunar spaces. (A, B: upper panel: magnification, ×100; lower panel: magnification, $\times 400)$ SMPC, stromal micropapillary component; AMPC, aerogenous micropapillary component.

Table 1 Antibodies

\begin{tabular}{|c|c|c|c|}
\hline Classification/Antibody & Clone & Dilution & Source \\
\hline \multicolumn{4}{|c|}{ Cellular adhesion molecules } \\
\hline E-cadherin & $\mathrm{NCH}-38$ & $1: 100$ & DakoCytomation, Carpinteria, CA, USA \\
\hline CD44 & DF1485 & $1: 400$ & Novocastra, Newcastle upon Tyne, UK \\
\hline Laminin $5 \gamma 2$ & $4 \mathrm{G} 1$ & $1: 50$ & DakoCytomation, Glostrup, Denmark \\
\hline \multicolumn{4}{|l|}{ Growth factor } \\
\hline VEGF-C & Polyclonal & $1: 50$ & Abcam, Cambridge, UK \\
\hline \multicolumn{4}{|c|}{ Apoptosis-associated proteins } \\
\hline $\mathrm{bc} / 2$ & 124 & $1: 50$ & DakoCytomation, Glostrup, Denmark \\
\hline p53 & DO-7 & Pre-diluted & Nichirei, Tokyo, Japan \\
\hline cleaved caspase-3 & Polyclonal & $1: 400$ & Cell signaling, Danvers, MA, USA \\
\hline \multicolumn{4}{|l|}{ Mucin-related proteins } \\
\hline MUC1 & Ma695 & 1:100 & Novocastra, Newcastle upon Tyne, UK \\
\hline MUC6 & CLH5 & 1:100 & Novocastra, Newcastle upon Tyne, UK \\
\hline \multicolumn{4}{|l|}{ Hypoxia induced protein } \\
\hline $\mathrm{HIF}-1 \alpha$ & EP1215Y & $1: 500$ & Abcam, Cambridge, UK \\
\hline \multicolumn{4}{|l|}{ Others } \\
\hline TTF-1 & $8 \mathrm{G} 7 \mathrm{G} 3 / 1$ & $1: 100$ & DakoCytomation, Carpinteria, CA, USA \\
\hline SP-A & PE10 & 1:100 & Dako, Kyoto, Japan \\
\hline Vimentin & V9 & Pre-diluted & DakoCytomation, Carpinteria, CA, USA \\
\hline Ki-67 & MIB-1 & $1: 50$ & Dako, Glostrup, Denmark \\
\hline LYVE1 & 15A5B2 & $1: 400$ & Oriental Yeast, Tokyo, Japan \\
\hline c-Met & EP1454Y & $1: 200$ & Abcam, Cambridge, UK \\
\hline Phospho-c-Met & Polyclonal & $1: 800$ & Stressgen, Ann Arbor, MI, USA \\
\hline
\end{tabular}

VEGF-C, vascular endothelial growth factor-C; HIF-1 $\alpha$, hypoxia induced factor $1-\alpha$; TF-1, thyroid transcription factor-1; SP-A, surfactant apoprotein A; LYVE1, lymphatic vessel endothelial hyaluronan receptor 1 . 
performed as follows. TMA recipient blocks were cut into 4- $\mu \mathrm{m}$-thick sections and mounted on silane-coated slides. HE staining was performed on initial sections to verify histology. The remaining sections were deparaffinized in xylene and dehydrated in a graded alcohol series, and endogenous peroxidase was blocked using 3\% hydrogen peroxide in absolute methyl alcohol. Heatinduced epitope retrieval was performed for $20 \mathrm{~min}$ at $95^{\circ} \mathrm{C}$ in $0.02 \mathrm{~mol} / \mathrm{L}$ citrate buffer ( $\mathrm{pH} \mathrm{6.0)}$ in samples fixed with $10 \%$ formalin if necessary. The slides were rinsed using deionized water and incubated with primary antibodies. They were then washed 3 times in phosphate-buffered saline and incubated with EnVision+ System-HRP (DAKO, Glostrup, Denmark). The reaction products were visualized using 3-3'-diaminobenzidine tetrahydrochloride, and sections were counterstained using hematoxylin. Additionally, a similar staining method was used for anti-podoplanin antibody (clone D2-40, pre-diluted; Ventana, Tucson, AZ, USA) to evaluate lymphatic permeation.

\section{Calculation of staining scores}

Immunostaining was scored based on staining intensity and percentage of positively stained cells, with 2 observers evaluating immunostained samples independently. When the observers gave different scores to immunostained samples, the slides were reviewed together under a multiheaded microscope until a consensus was reached. Sections were classified by staining intensity as negative (total absence of staining), 1+ (weak staining), 2 + (moderate staining), or 3+ (strong staining). Staining scores were calculated by multiplying the percentage of positive tumor cells per section $(0-100 \%)$ by the staining intensity; scores obtained ranged from 0 to 300. Expression of p53, cleaved caspase-3, and Ki-67 were determined by counting 300 tumor cells under a high power field $(\times 400)$ and results are shown as the percentage of positive cells.

\section{Mutation analysis}

Mutation analyses of EGFR gene exons 19 and 21 and KRAS gene codons 12 and 13 were performed using loop-hybrid mobility shift assays and gene sequencing procedures described elsewhere [13].

\section{Statistical analysis}

All calculations were performed using SPSS software (Dr. SPSS II for Windows Standard version 11.0; SPSS Inc., Chicago, IL, USA). The Chi-square for independence or Fisher's exact probability test was performed to analyze differences in patient characteristics between the 2 groups. The Fisher's exact probability test was performed if there were 5 or fewer observations in a group. For univariate analysis, all cumulative survival was estimated using the Kaplan-Meier method, and differences in variables were calculated using the log-rank test. Multivariate regression analysis was conducted according to the Cox proportional hazard model. The Mann-Whitney $U$ test was used to compare staining scores. Differences were considered significant when the $P$ value was less than 0.05 .

\section{Results}

\section{Clinicopathological characteristics of patients with SMPC}

Figure 2 shows a Venn diagram of the relationship between the micropapillary component sets in the 559 patients examined in this study. SMPC was observed in 19 patients (3.4\%) and AMPC in 99 (17.7\%) patients. A mixture of SMPC and AMPC was observed in 14 patients, pure SMPC without AMPC in 5 patients, and pure AMPC without SMPC in 85 patients. A micropapillary pattern was observed in $50-100 \%$ in 2 SMPC tumor and less than 50\% in 17 SMPC tumors. No SMPC(+) tumors were completely replaced by SMPC. Clinicopathological characteristics of patients with SMPC (+) and SMPC(-) tumors are summarized in Table 2. Patients with $\mathrm{SMPC}(+)$ tumors were significantly found to be at a more advanced stage, larger than $30 \mathrm{~mm}$ in diameter, and have more frequent lymph node metastasis compared to those with SMPC(-) tumors. Pleural, lymphatic, and vascular invasion were observed more often in patients with $\mathrm{SMPC}(+)$ tumors than in those with SMPC(-) tumors. (68\% vs. $17 \%, P<$ $0.001 ; 74 \%$ vs. $15 \%, P<0.001 ; 74 \%$ vs. $22 \%, P<0.001$, respectively). No significant differences in age, gender,

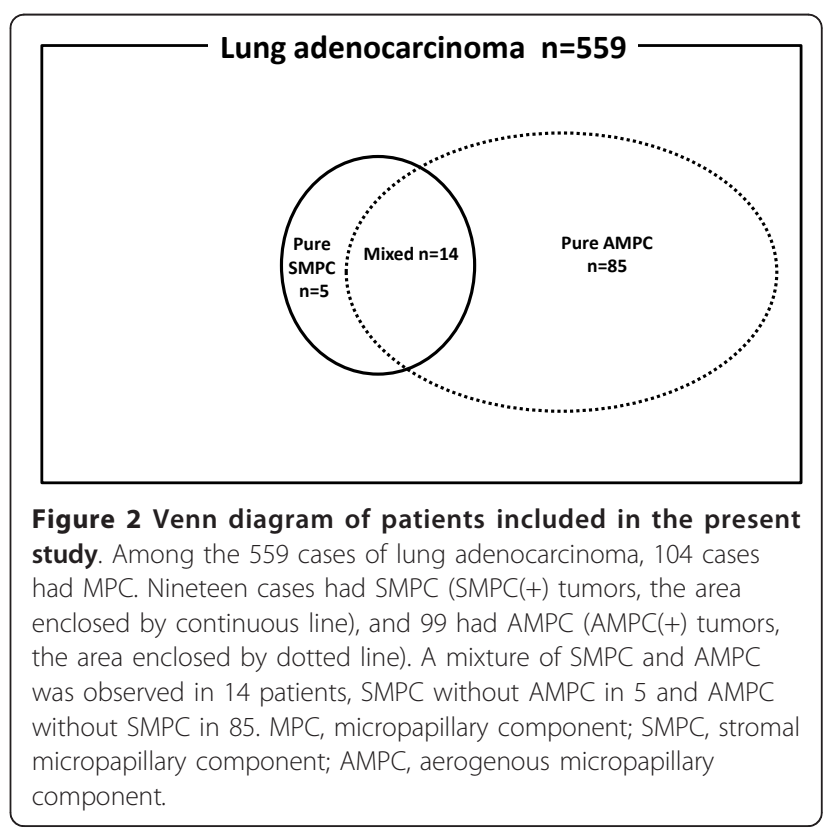


Table 2 Clinicopathological characteristics of patients with SMPC

\begin{tabular}{|c|c|c|c|c|c|c|c|}
\hline & all & $\%$ & & SM & IPC & & $P$ value \\
\hline & & & $(-)$ & $\%$ & $(+)$ & $\%$ & \\
\hline No. & 559 & & 540 & 97 & 19 & 3 & \\
\hline Age & & & & & & & \\
\hline Median & 67 & & 67 & & 67 & & $0.219^{*}$ \\
\hline Range & $23-87$ & & $23-87$ & & $40-76$ & & \\
\hline Gender & & & & & & & \\
\hline Female & 288 & 52 & 282 & 52 & 6 & 32 & $0.077^{* *}$ \\
\hline Male & 271 & 48 & 258 & 48 & 13 & 68 & \\
\hline Smoking status & & & & & & & \\
\hline Nonsmoker & 284 & 51 & 276 & 51 & 8 & 42 & $0.596^{* *}$ \\
\hline Smoker & 275 & 49 & 264 & 49 & 11 & 58 & \\
\hline Bl Average & 369 & & 364 & & 502 & & \\
\hline Tumor size & & & & & & & \\
\hline Average(mm) & 25 & & 25 & & 35 & & \\
\hline Range(mm) & $5-140$ & & $5-140$ & & $15-75$ & & \\
\hline$<30 \mathrm{~mm}$ & 396 & 71 & 388 & 72 & 9 & 47 & $<0.001^{*}$ \\
\hline$\geq 30 \mathrm{~mm}$ & 163 & 29 & 152 & 28 & 10 & 53 & \\
\hline Pathological stage & & & & & & & \\
\hline IA & 363 & 65 & 360 & 67 & 4 & 21 & $<0.001^{* *}$ \\
\hline $\mathrm{IB}$ & 95 & 17 & 88 & 16 & 6 & 32 & \\
\hline$\| A$ & 36 & 6 & 31 & 6 & 5 & 26 & \\
\hline$\| B$ & 13 & 2 & 13 & 2 & 0 & 0 & \\
\hline$\| I A$ & 42 & 8 & 39 & 7 & 3 & 16 & \\
\hline$\geq \| \mathrm{IIB}$ & 10 & 2 & 9 & 2 & 1 & 5 & \\
\hline Lymph node metas & asis & & & & & & \\
\hline NX & 69 & 12 & 68 & 13 & 1 & 5 & \\
\hline NO & 420 & 75 & 409 & 75 & 11 & 58 & $0.002^{* *}$ \\
\hline$\geq N 1$ & 70 & 13 & 63 & 12 & 7 & 21 & \\
\hline Pleural invasion & & & & & & & \\
\hline Negative & 452 & 80 & 446 & 83 & 6 & 32 & $<0.001^{* *}$ \\
\hline Positive & 107 & 20 & 94 & 17 & 13 & 68 & \\
\hline Lymphatic invasion & & & & & & & \\
\hline Negative & 466 & 83 & 461 & 85 & 5 & 26 & $<0.001^{* *}$ \\
\hline Positive & 93 & 17 & 79 & 15 & 14 & 74 & \\
\hline Vascular invasion & & & & & & & \\
\hline Negative & 427 & 76 & 422 & 78 & 5 & 26 & $<0.001^{* *}$ \\
\hline Positive & 132 & 24 & 118 & 22 & 14 & 74 & \\
\hline
\end{tabular}

* Mann-Whitney's U test

** Chi-square for independence test

No., number of patients; Bl, Brinkman index = (number of cigarettes per day) $\times$ (duration of years); SMPC, stromal micropapillary component; AMPC, aerogeneous micropapillary component

or smoking status were observed between patients with SMPC(+) and SMPC(-) tumors.

\section{Survival analysis}

Among all stage patients, median follow-up time was 654 days (range, 33-1512 days) in SMPC(-) tumors, 240 days (range, 28-661 days) in SMPC(+) tumors, 664 days (range, 28-1512 days) in AMPC(-) tumors, and 467 days (range, 36-1412 days) in AMPC(+) tumors. Among the stage I patients, median follow-up time was 767 days (range, 59-1343 days) in SMPC(-) tumors, 192 days (range, 227-485 days) in SMPC(+) tumors, 767 days (range, 59-1343 days) in AMPC(-) tumors, and 836 days (range, 140-1233 days) in AMPC(+) tumors. Recurrence occurred in 28 of 559 cases. SMPC $(+)$ tumors recurred in 4 of 19 in all stage and in 2 of 10 in p-stage I, and AMPC(+) tumors recurred in 8 of 99 cases and 4 of 69 cases, respectively. In all stage, disease-free survival (DFS) of patients with SMPC(+) tumors was significantly poorer than that in patients with SMPC(-) tumors (Figure 3A, $\mathrm{P}<0.001$ ); the same result was observed in patients with $\mathrm{AMPC}(+)$ and $\mathrm{AMPC}(-)$ tumors (Figure $3 \mathrm{~B}, \mathrm{P}=0.045$,). In p-stage I patients, DFS of those with $\mathrm{SMPC}(+)$ tumors showed significantly poorer outcome than that of patients with SMPC(-) tumors (Figure 3C, $\mathrm{P}<0.001)$; the same result was observed between patients with $\mathrm{AMPC}(+)$ and $\mathrm{AMPC}(-)$ tumors (Figure 3D, $\mathrm{P}=0.023$ ).

In univariate analysis, high pathological stage $(P<$ $0.001)$, pleural invasion $(P<0.001)$, lymphatic invasion $(P$ $<0.001)$, vascular invasion $(P<0.001), \operatorname{SMPC}(+)(P<$ $0.001)$, and AMPC $(+)$ tumors $(P=0.045)$ showed an unfavorable influence on survival for all stage, and pleural invasion $(P<0.001)$, lymphatic invasion $(P<0.001)$, vascular invasion $(P<0.001), \operatorname{SMPC}(+)(P<0.001)$, and AMPC $(+)$ tumors $(P=0.023)$ showed an unfavorable influence on survival for p-stage I (Table 3,4$)$. In multivariate analysis, pathological stage $(P=0.028)$, lymphatic invasion $(P=0.009)$, and vascular invasion $(P=0.011)$ were identified as significant independent prognostic factors for all stage (Table 3$)$. Though not observed for all stage, the presence of SMPC $(+)$ tumors $(P=0.035)$ was identified as a significant independent prognostic factor for $\mathrm{p}$-stage $\mathrm{I}$, as well as lymphatic invasion $(P=0.020)$ and vascular invasion $(P=0.049)$ (Table 4$)$. The presence of $\mathrm{AMPC}(+)$ tumors was not a significant prognostic factor for all stage or p-stage I.

\section{Immunohistochemical findings}

We evaluated immunohistochemical profiles of SMPC, AMPC, and nMPC. These lesions were evaluated in TMAs for 33 cases, including $19 \mathrm{SMPC}(+)$ tumors and 14 pure AMPC tumors. The latter 14 tumors were selected from 85 pure AMPC tumors according to operation date, patient age, gender, and smoking status to match clinical background factors between SMPC and AMPC. nMPC was generally included in TMA cores of SMPC and AMPC. The total number of TMA was 19 SMPC and 28 AMPC. Staining scores are summarized in Table 5.

In cellular adhesion molecules, E-cadherin staining scores in patients with SMPC, AMPC, and $\mathrm{nMPC}$ were 

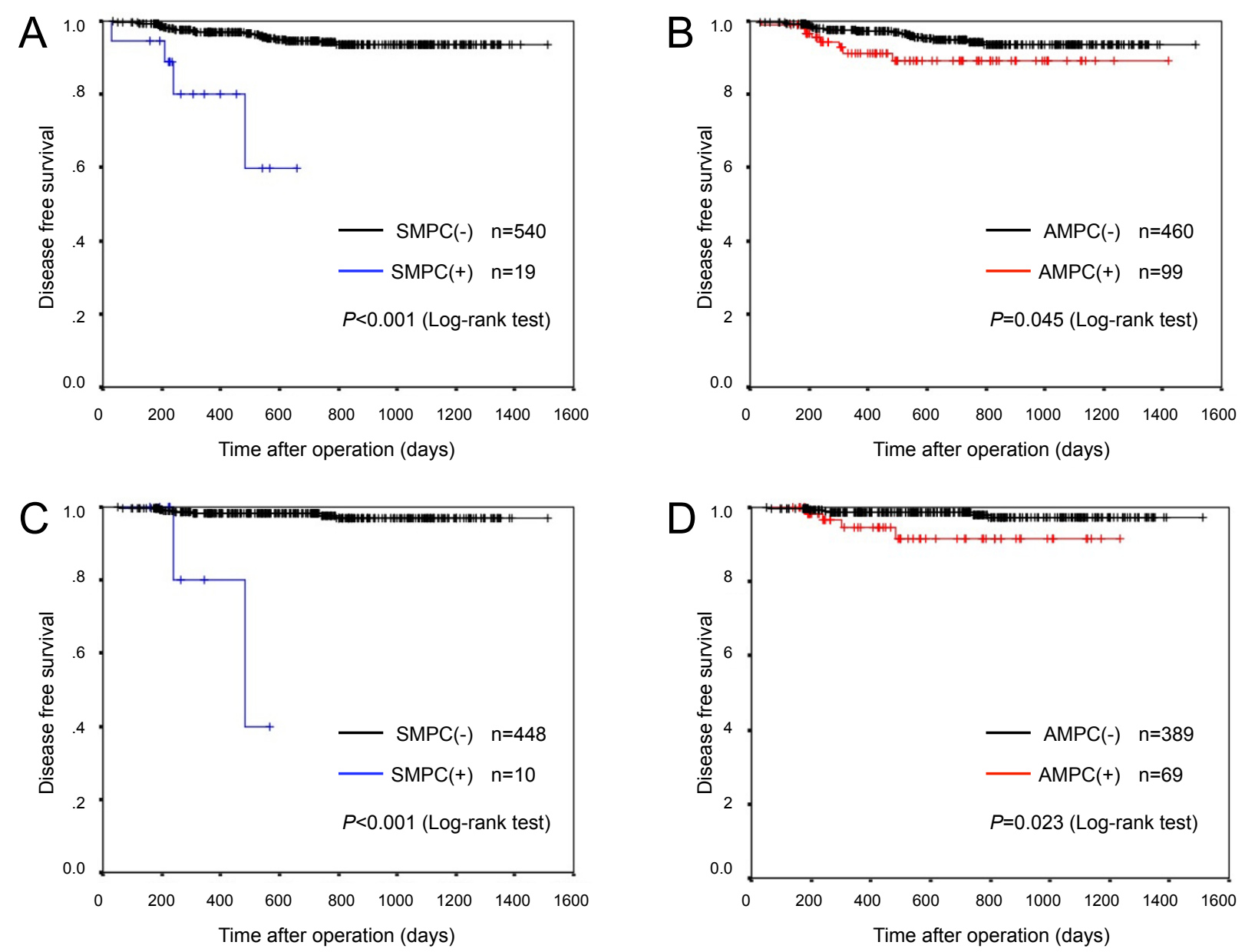

Figure 3 Cumulative disease-free survival rates of patients according to presence of SMPC and AMPC. A, B are cumulative disease-free survival rates in all stage, and C, D are that of in p-stage I. Cumulative disease-free survival rates stratified by presence of SMPC are shown in A and $C$, and those stratified by presence of AMPC are shown in B and D. In all stage and in p-stage I, SMPC (+) tumors and AMPC(+) tumors had significantly poorer outcomes. Outcomes of $\mathrm{SMPC}(+)$ tumors were more significantly negative than those of AMPC(+) tumors. SMPC, stromal micropapillary component; AMPC, aerogenous micropapillary component.

215.3, 143.9, and 187.1, respectively, and although the differences were not significant between patients with SMPC or nMPC and between patients with AMPC or nMPC $(P=0.312,0.127$, respectively), staining scores of SMPC were significantly higher than those for patients with AMPC $(P=0.020)$ (Figure 4A-C). CD44 staining scores in SMPC, AMPC, and nMPC were 60.8, 205.9, and 141.3, respectively. The CD44 expression level in SMPC was significantly lower than in AMPC $(P<$ $0.001)$ and significantly higher than that in $\mathrm{nMPC}$ lesions $(P=0.015)$ (Figure 4D-F).

For other antibodies, staining scores of surfactant apoprotein A (SP-A) in the SMPC, AMPC, and $\mathrm{nMPC}$ were $45.2,82.6$, and 123.2, respectively, and although the difference was not significant between AMPC and nMPC $(P=0.203)$, the staining score in SMPC was significantly lower than those in nMPC $(P=0.024)$ (Figure 4G-I).
Similarly, staining scores of phospho-c-Met in SMPC, AMPC, and $\mathrm{nMPC}$ were $34.2,50.5,88.0$, respectively, and staining scores in SMPC were significantly lower than those in nMPC (Figure 4J-L).

\section{Mutation analysis}

Mutation analysis was performed in 33 patients for whom TMAs were constructed for immunohistochemical analysis. Table 6 summarizes the results of the mutation analysis. Although no cases examined possessed the KRAS mutations, EGFR mutations were detected in 20 cases (61\%): 14 in patients with SMPC(+) tumors (74\%) and 6 in patients with SMPC(-) tumors (43\%). There was no significant association between the existence of SMPC and EGFR mutations. Among the 20 cases with EGFR mutations, 7 had deletions at exon 19, 13 had a point mutation at exon 21 , and there were no 
Table 3 Impact of potential prognostic factors on DFS of patients of lung adenocarcinoma in all stage by univariate and multivariate analysis

\begin{tabular}{|c|c|c|c|c|c|c|}
\hline & \multirow[t]{2}{*}{ No. } & \multirow[t]{2}{*}{$\%$} & \multirow{2}{*}{$\begin{array}{c}\text { Univariate } \\
\text { analysis }\end{array}$} & \multicolumn{3}{|c|}{ Multivariate analysis } \\
\hline & & & & $\begin{array}{c}\text { Hazard } \\
\text { ratio }\end{array}$ & $95 \% \mathrm{Cl}$ & $\begin{array}{c}P \\
\text { value }\end{array}$ \\
\hline Total & 559 & & & & & \\
\hline \multicolumn{7}{|l|}{ Age } \\
\hline$<65$ & 213 & 38 & 0.388 & 1.000 & & \\
\hline$\geq 65$ & 346 & 62 & & 1.933 & $0.849-4.402$ & 0.116 \\
\hline \multicolumn{7}{|l|}{ Gender } \\
\hline Female & 288 & 52 & 0.768 & 1.000 & & \\
\hline Male & 271 & 48 & & 0.807 & $0.232-2.803$ & 0.735 \\
\hline \multicolumn{7}{|l|}{ Smoking status } \\
\hline $\begin{array}{l}\text { Non- } \\
\text { smoker }\end{array}$ & 284 & 49 & 0.560 & 1.000 & & \\
\hline Smoker & 275 & 51 & & 1.164 & $0.342-3.956$ & 0.808 \\
\hline \multicolumn{7}{|l|}{ Tumor size } \\
\hline$<30 \mathrm{~mm}$ & 396 & 71 & 0.059 & 1.000 & & \\
\hline$\geq 30 \mathrm{~mm}$ & 163 & 29 & & 0.819 & $0.338-1.985$ & 0.658 \\
\hline \multicolumn{7}{|l|}{$\begin{array}{l}\text { Pathological } \\
\text { stage }\end{array}$} \\
\hline । & 458 & 82 & $<0.001$ & 1.000 & & \\
\hline II, III, IV & 101 & 18 & & 2.768 & $1.113-6.884$ & 0.028 \\
\hline \multicolumn{7}{|l|}{ Pleural invasion } \\
\hline Negative & 452 & 81 & $<0.001$ & 1.000 & & \\
\hline Positive & 107 & 19 & & 0.848 & $0.345-2.083$ & 0.719 \\
\hline \multicolumn{7}{|l|}{$\begin{array}{l}\text { Lymphatic } \\
\text { invasion }\end{array}$} \\
\hline Negative & 466 & 83 & $<0.001$ & 1.000 & & \\
\hline Positive & 93 & 17 & & 3.430 & $1.363-8.634$ & 0.009 \\
\hline \multicolumn{7}{|l|}{$\begin{array}{l}\text { Vascular } \\
\text { invasion }\end{array}$} \\
\hline Negative & 427 & 76 & $<0.001$ & 1.000 & & \\
\hline Positive & 132 & 24 & & 3.309 & $1.312-8.350$ & 0.011 \\
\hline \multicolumn{7}{|l|}{ SMPC } \\
\hline Negative & 540 & 97 & $<0.001$ & 1.000 & & \\
\hline Positive & 19 & 3 & & 1.871 & $0.528-6.630$ & 0.332 \\
\hline \multicolumn{7}{|l|}{ AMPC } \\
\hline Negative & 460 & 83 & 0.045 & 1.000 & & \\
\hline Positive & 99 & 17 & & 1.132 & $0.450-2.845$ & 0.792 \\
\hline
\end{tabular}

DFS, disease free survival; No., number of patients; SMPC, stromal micropapillary component; AMPC, aerogeneous micropapillary component; $\mathrm{Cl}$, confidence interval.

multiple mutations. Among the 13 cases with a point mutation at exon 21, 12 had an L858R mutation and one had an L861Q mutation.

\section{Discussion}

The present study revealed the incidence of SMPC(+) lung adenocarcinoma in consecutive surgical cases to be $3.4 \%$, which is lower than that of $\mathrm{AMPC}(+)$ lung
Table 4 Impact of potential prognostic factors on DFS of patients of lung adenocarcinoma in p-stage I by univariate and multivariate analysis

\begin{tabular}{|c|c|c|c|c|c|c|}
\hline & \multirow[t]{2}{*}{ No. } & \multirow[t]{2}{*}{$\%$} & \multirow{2}{*}{$\begin{array}{c}\begin{array}{c}\text { Univariate } \\
\text { Analysis }\end{array} \\
P \text { value }\end{array}$} & \multicolumn{3}{|c|}{ Multivariate analysis } \\
\hline & & & & $\begin{array}{c}\text { Hazard } \\
\text { ratio }\end{array}$ & $95 \% \mathrm{Cl}$ & $\begin{array}{c}P \\
\text { value }\end{array}$ \\
\hline Total & 458 & & & & & \\
\hline \multicolumn{7}{|l|}{ Age } \\
\hline$<65$ & 172 & 38 & 0.394 & 1.000 & & \\
\hline$\geq 65$ & 286 & 62 & & 2.191 & $0.474-10.131$ & 0.316 \\
\hline \multicolumn{7}{|l|}{ Gender } \\
\hline Female & 249 & 54 & 0.063 & 1.000 & & \\
\hline Male & 209 & 46 & & 0.157 & $0.014-1.787$ & 0.136 \\
\hline \multicolumn{7}{|l|}{ Smoking status } \\
\hline $\begin{array}{l}\text { Non- } \\
\text { smoker }\end{array}$ & 248 & 54 & 0.204 & 1.000 & & \\
\hline Smoker & 210 & 46 & & 0.768 & $0.117-5.052$ & 0.784 \\
\hline \multicolumn{7}{|l|}{ Tumor size } \\
\hline$<30 \mathrm{~mm}$ & 358 & 78 & 0.264 & 1.000 & & \\
\hline$\geq 30 \mathrm{~mm}$ & 100 & 22 & & 0.304 & $0.037-2.504$ & 0.268 \\
\hline \multicolumn{7}{|l|}{$\begin{array}{l}\text { Pleural } \\
\text { invasion }\end{array}$} \\
\hline Negative & 402 & 88 & $<0.001$ & 1.000 & & \\
\hline Positive & 56 & 12 & & 1.519 & $0.328-7.040$ & 0.593 \\
\hline \multicolumn{7}{|l|}{$\begin{array}{l}\text { Lymphatic } \\
\text { invasion }\end{array}$} \\
\hline Negative & 415 & 91 & $<0.001$ & 1.000 & & \\
\hline Positive & 43 & 9 & & 5.016 & $1.295-19.434$ & 0.020 \\
\hline \multicolumn{7}{|l|}{$\begin{array}{l}\text { Vascular } \\
\text { invasion }\end{array}$} \\
\hline Negative & 390 & 85 & $<0.001$ & 1.000 & & \\
\hline Positive & 68 & 15 & & 4.494 & $1.006-20.081$ & 0.049 \\
\hline \multicolumn{7}{|l|}{ SMPC } \\
\hline Negative & 448 & 98 & $<0.001$ & 1.000 & & \\
\hline Positive & 10 & 2 & & 9.028 & $1.164-70.031$ & 0.035 \\
\hline \multicolumn{7}{|l|}{ AMPC } \\
\hline Negative & 389 & 98 & 0.023 & 1.000 & & \\
\hline Positive & 69 & 2 & & 1.825 & $0.378-8.808$ & 0.454 \\
\hline
\end{tabular}

DFS, disease free survival; No., number of patients; SMPC, stromal micropapillary component; AMPC, aerogeneous micropapillary component; $\mathrm{Cl}$, confidence interval.

adenocarcinoma (17.7\%). In non-pulmonary organs, the incidence of invasive micropapillary carcinoma was reported to be $7 \%$ in breast carcinoma [14], $0.9 \%$ in urinary bladder cancer [15], and $9.4 \%$ in colon cancer [16]. Generally, invasive micropapillary carcinomas occur infrequently in any organ.

Prognosis of lung adenocarcinoma with MPC has been reported to be worse and have the potential for high malignancy $[17,18]$, but no studies have separately evaluated SMPC and AMPC. We showed that SMPC(+) 
Table 5 Staining Scores in SMPC, AMPC and nMPC lesions

\begin{tabular}{|c|c|c|c|}
\hline Classification/Antibody & SMPC & AMPC & nMPC \\
\hline \multicolumn{4}{|l|}{ Cellular adhesion molecules } \\
\hline E-cadherin & $215.3^{*}$ & 143.9 & 187.1 \\
\hline CD44 & $60.8^{\ddagger}$ & $205.9^{\natural}$ & 141.3 \\
\hline Laminin $5 \gamma 2$ & 69.4 & 36.9 & 60.3 \\
\hline \multicolumn{4}{|l|}{ Growth factor } \\
\hline VEGF-C & 294.4 & 296.4 & 282.1 \\
\hline \multicolumn{4}{|c|}{ Apoptosis-associated proteins } \\
\hline$b c \mid 2$ & 13.2 & 11.1 & 21.8 \\
\hline $\mathrm{p} 53^{\S}$ & 36.4 & 26.1 & 45.0 \\
\hline cleaved caspase- $3^{\S}$ & 0.3 & 0.2 & 0.4 \\
\hline \multicolumn{4}{|l|}{ Mucin-related proteins } \\
\hline MUC1 & 169.7 & 182.5 & 202.1 \\
\hline MUC6 & 0.0 & 0.0 & 0.0 \\
\hline \multicolumn{4}{|l|}{ Hypoxia induced protein } \\
\hline $\mathrm{HIF}-1 \alpha$ & 1.8 & 2.4 & 2.9 \\
\hline \multicolumn{4}{|l|}{ Others } \\
\hline TTF-1 & 267.9 & 289.3 & 248.6 \\
\hline SP-A & $45.2^{\ddagger}$ & 82.6 & 123.2 \\
\hline Vimentin & 112.1 & 117.9 & 72.1 \\
\hline $\mathrm{Ki}-67^{\S}$ & 22.6 & 16.9 & 16.2 \\
\hline LYVE1 & 98.9 & 107.2 & 101.9 \\
\hline c-Met & 217.2 & 253.8 & 211.4 \\
\hline Phospho-c-Met & $34.2^{\ddagger}$ & 50.0 & 88.0 \\
\hline
\end{tabular}

SMPC, stromal micropapillary component; AMPC, aerogeneous micropapillary component; nMPC, non-micropapillary component; VEGF-C, vascular endothelial growth factor-C; HIF-1 $\alpha$, hypoxia induced factor $1-\alpha$; TTF-1, thyroid transcription factor-1; SP-A, surfactant apoprotein A; LYVE1, lymphatic vessel endothelial hyaluronan receptor 1.

$\S$ Positivity rate of positive tumor cells in 300 tumor cells (percentage).

* The difference in staining scores between SMPC and AMPC is statistically significant $(P=0.020)$.

₹ The differences in staining scores between SMPC and $\mathrm{nMPC}$ are statistically significant for CD44 $(P=0.011)$, and SP-A $(P=0.024)$ and phospho-c-Met $(P=$ 0.011) expression.

I The difference in staining scores between AMPC and nMPC is statistically significant $(P=0.015)$.

tumors as well as AMPC(+) tumors are associated with several biological factors including tumor size, lymph node metastasis, advanced stage disease, and pleural and lymphovascular invasion. Univariate analysis also revealed the presence of SMPC and AMPC as a significant predictor of unfavorable outcome. However, the most remarkable finding was observed in multivariate analysis: among the patients in p-stage I, patients with not AMPC but SMPC showed a significantly poorer DFS than those without MPC. We used immunohistochemistry with monoclonal antibody D2-40 against lymphatic endothelium in TMA specimens and found that lymphatic vessels are involved within SMPC areas in 4 (21\%) of $19 \mathrm{SMPC}(+)$ tumors (data not shown). When compared with $\mathrm{AMPC}(+)$ tumors, SMPC $(+)$ tumors significantly more often showed pleural, lymphatic, and vascular invasion than $\mathrm{AMPC}(+)$ tumors $(68 \%$ vs. $33 \%$, $P=0.004 ; 74 \%$ vs. $30 \%, P<0.001 ; 74 \%$ vs. $41 \%, P=$ 0.010 , respectively). Therefore, these data suggest that a strong association between SMPC(+) tumors and pleural and lymphovascular invasion may in part explain their aggressive behavior.

Moreover, we investigated the immunohistochemical differences between SMPC and AMPC. In the study, we observed high E-cadherin expression and low CD44 expression in SMPC. Phospho-c-Met expression generally decreases in SMPC to a greater extent than in AMPC. Recently, it has been suggested that E-cadherin repression and CD44 expression are associated with the epithelial-mesenchymal transition (EMT), which was thought to lead to tumor invasion $[19,20]$. Additionally, Elliot et al. reported that hepatocyte growth factor (HGF) and c-Met signaling promotes EMT in breast cancer [21], and Orian-Rousseau et al. reported that CD44 is strictly required for c-Met activation by HGF in human carcinoma [22]. Consistent with these data, EMT may not occur in SMPC despite its existence in the stroma, or invasion of SMPC may occur through a different invasion mechanism from EMT. Our immunohistochemical findings of SMPC showed lower expression of SP-A than that of nMPC. Many studies have reported that SP-A deletion is correlated with patient survival, and reduced SP-A in MPC may be an excellent indicator for poor prognosis in small-size lung adenocarcinoma $[23,24]$. Reduced SP-A may contribute to an unfavorable outcome of SMPC $(+)$ tumors.

Some studies have reported a significant association between the presence of MPC and EGFR mutations and effectiveness of EGFR tyrosine kinase inhibitor (EGFRTKI) for MPC(+) tumors [25-28]. Since SMPC of lung adenocarcinoma may be associated with a high incidence of EGFR mutations, EGFR-TKI may be effective against $\mathrm{SMPC}(+)$ tumors. Patients with these pathological features of lung adenocarcinoma may benefit from EGFR-TKI as postoperative chemotherapy or first-line chemotherapy of relapsed lung adenocarcinoma.

In conclusion, we observed $\mathrm{SMPC}(+)$ adenocarcinoma. The incidence of SMPC(+) tumors is low, and SMPC(+) tumors have a different prognostic impact compared to AMPC (+) tumors. Particularly for the early stage tumors, $\mathrm{SMPC}(+)$ tumors have different pathobiological characteristics from AMPC(+) tumors, and SMPC(+) tumors frequently contain the EGFR mutation. Therefore, it is important to determine the presence of SMPC in lung adenocarcinoma, particularly p-stage I tumors, and the presence of SMPC should be noted in a pathology report to alert the clinician to the possibility of poor prognosis. 


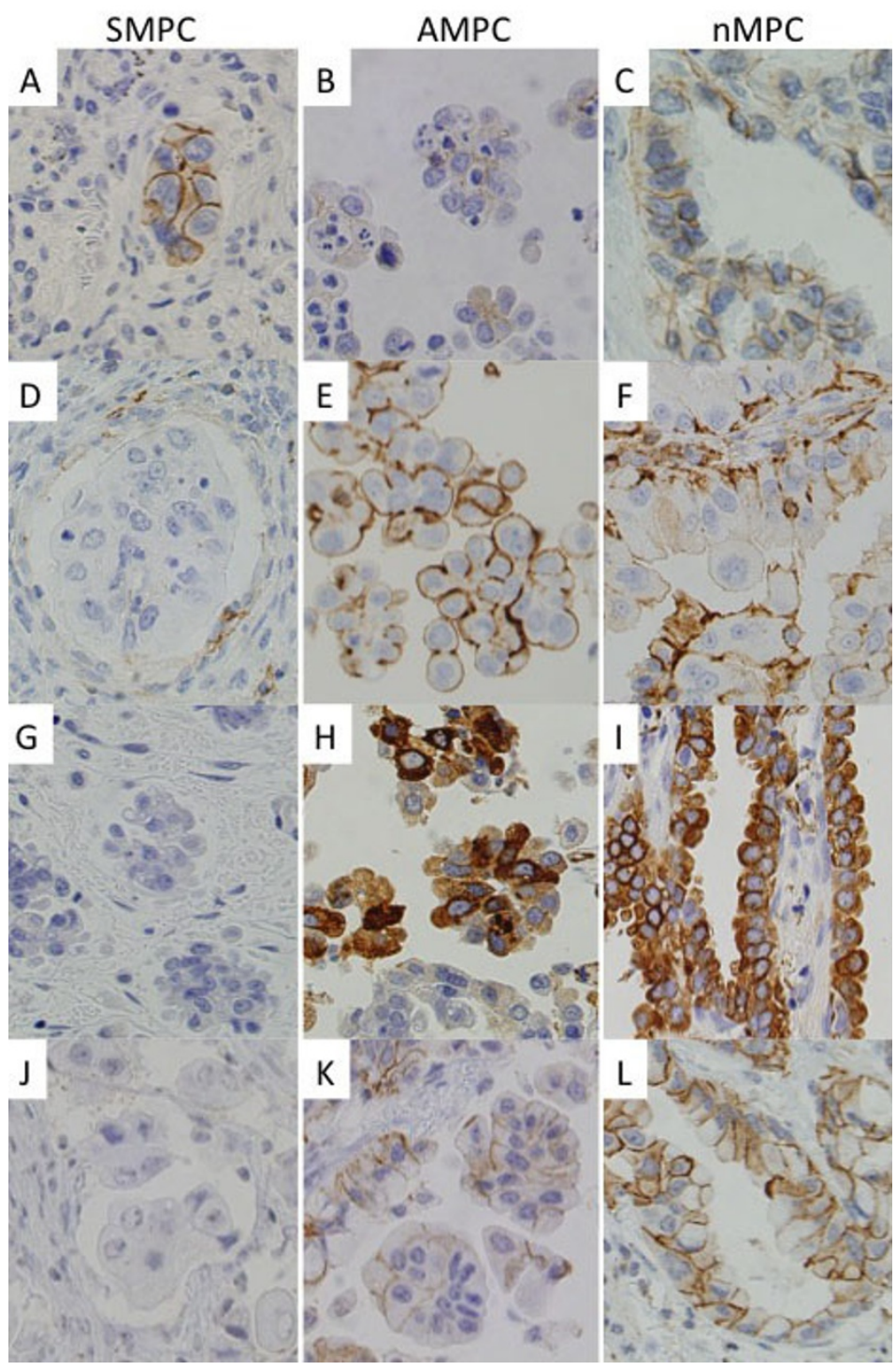

Figure 4 Photomicrographs of immunohistochemistry. E-cadherin (A-C); CD44 (D-F); SP-A (G-I); Phospho-C-Met (J-L). Compared with AMPC, increased E-cadherin and decreased CD44 membrane immunostaining were found in SMPC. Moreover, SP-A cytoplasm and Phospho-CMet membrane immunostaining were decreased in SMPC (×400). SMPC, stromal micropapillary component, left panels; AMPC, aerogenous micropapillary component, middle panels; nMPC, non-micropapillary component, right panels.

Table 6 Mutation analysis

\begin{tabular}{|c|c|c|c|c|c|c|c|}
\hline & total & $\%$ & SMPC(+) cases & $\%$ & SMPC(-) cases & $\%$ & $P$ value \\
\hline No. & 33 & & 19 & & 14 & & \\
\hline \multicolumn{8}{|l|}{ EGFR mutation } \\
\hline Negative & 13 & 39 & 5 & 26 & 8 & 57 & $0.076^{*}$ \\
\hline Positive & 20 & 61 & 14 & 74 & 6 & 43 & \\
\hline ex19 & 7 & $35^{\S}$ & 5 & $36^{\S}$ & 2 & $33^{\S}$ & $0.664^{*}$ \\
\hline ex21 & 13 & $65^{\S}$ & 9 & $64^{\S}$ & 4 & $67^{\S}$ & \\
\hline \multicolumn{8}{|l|}{ KRAS mutation } \\
\hline Negative & 33 & 100 & 19 & 100 & 14 & 100 & - \\
\hline Positive & 0 & 0 & 0 & & 0 & & \\
\hline
\end{tabular}

No., number of patients; EGFR, epidermal growth factor receptor; SMPC, stromal micropapillary component

* Fisher's exact probability test

$\S$ Rate of positive cases at ex19 and 21 in EGFR mutation positive cases, respectively. 


\section{List of abbreviations}

AMPC: aerogenous micropapillary component; SMPC: stromal micropapillary component; MPC: micropapillary component; TMA: tumor tissue microarray; DFS: disease-free survival.

\section{Acknowledgements}

The authors thank Ms. Yoshihara for TMA synthesis.

This article was supported by Kanagawa Cancer Research Fund and Grant for Collaboration between Hospital and Research Institute, Kanagawa Cancer Center.

\section{Author details}

'Department of Pathology, Kanagawa Cancer Center, 1-1-2 Nakao, Asahi-ku, Yokohama, Kanagawa, 241-0815, Japan. 'Department of Thoracic Oncology, Kanagawa Cancer Center, 1-1-2 Nakao, Asahi-ku, Yokohama, Kanagawa, 241 0815, Japan. ${ }^{3}$ Molecular Pathology and Genetics Division, Kanagawa Cancer Center Research Institute, 1-1-2 Nakao, Asahi-ku, Yokohama, Kanagawa, $241-$ 0815, Japan. ${ }^{4}$ Cancer Prevention and Cancer Control Division, Kanagawa Cancer Center Research Institute, 1-1-2 Nakao, Asahi-ku, Yokohama, Kanagawa, 241-0815, Japan. ${ }^{5}$ Division of Clinical Oncology and Respiratory Medicine, Department of Internal Medicine, Shimane University Faculty of Medicine, 89-1 Enya-cho, Izumo, Shimane, 693-8501, Japan.

\section{Authors' contributions}

$\mathrm{MO}$ and TY designed the study, performed clinical and pathological investigation, and wrote the drafts. YS and YM participated in pathological and genetical investigation. NO participated in statistical investigation. SO performed the histological and immunohistochemical evaluation. $\mathrm{CH}$ assisted the clinical investigation. HN participated in managing and operating the patients. YK assisted the pathological investigation. KY participated in collecting clinical data and images. TI participated in its design and coordination and helped to draft the manuscript. All authors read and approved the final manuscript.

\section{Competing interests}

The authors declare that they have no competing interests.

Received: 3 November 2011 Accepted: 6 January 2012 Published: 6 January 2012

\section{References}

1. Travis WD, et al: International Association for the Study of Lung Cancer/ American Thoracic Society/European Respiratory Society international multidisciplinary classification of lung adenocarcinoma. J Thorac Oncol 2011, 6:244-285.

2. Yoshizawa A, Motoi N, Riely GJ, Sima CS, Gerald WL, Kris MK, Park BJ, Rusch WW, Travis WD: Impact of proposed IASLC/ATS/ERS classification of lung adenocarcinoma: prognostic subgroups and implications for further revision of staging based on analysis of 514 stage I cases. Mod Pathol 2011, 24:653-664.

3. Amin MB, Tamboli P, Merchant SH, Ordóñez NG, Ro J, Ayala AG, Ro JY: Micropapillary component in lung adenocarcinoma: a distinctive histologic feature with possible prognostic significance. Am J Surg Pathol 2002, 26:358-364.

4. Miyoshi T, Satoh Y, Okumura S, Nakagawa K, Shirakusa T, Tsuchiya E, Ishikawa Y: Early-stage lung adenocarcinoma with micropapillary pattern, a distinct pathologic marker for a significantly poor prognosis. Am J Surg Pathol 2003, 27:101-109.

5. Maeda R, Isowa N, Onuma H, Miura H, Harada T, Touge H, Tokuyasu $H_{\text {, }}$ Kawasaki Y: Lung adenocarcinomas with micropapillary components. Gen Thorac Cardiovasc Surg 2009, 57:534-539.

6. Nagano T, Ishii G, Nagai K, Ito T, Kawase A, Takahashi K, Nishimura Y, Noshiwaki Y, Ochiai A: Structural and biological properties of a papillary component generating a micropapillary component in lung adenocarcinoma. Lung Cancer 2010, 67:282-289.

7. Roh MS, Lee Jl, Choi PJ, Hong YS: Relationship between micropapillary component and micrometastasis in the regional lymph nodes of patients with stage I lung adenocarcinoma. Histopathology 2004, 45:580-586.

8. Kamiya K, Hayashi Y, Douguchi J, Hashiguchi A, Yamada T, Izumi Y, Watanabe M, Kawamura M, Horinouchi H, Shimada N, Kobayashi K,
Sakamoto M: Histopathological features and prognostic significance of the micropapillary pattern in lung adenocarcinoma. Mod Pathol 2008, 21:992-1001.

9. Ohe M, Yokose T, Sakuma Y, Osanai S, Hasegawa C, Washimi K, Nawa K, Woo T, Hamanaka R, Nakayama H, Kameda K, Yamada K, Isobe T: Stromal micropapillary pattern predominant lung adenocarcinoma - A report of two cases. Diagn Pathol 2011, 6:92.

10. Kuroda N, Hamaguchi N, Takeuchi E, Ohara M, Hirouchi T, Mizuno K: Lung adenocarcinoma with a micropapillary pattern: a clinicopathological study of 25 cases. APMIS 2006, 114:381-385.

11. In TNM classification of malignant tumors.. seventh edition. Edited by: Sobin LH, Gospodarowicz MK, Wittekind C. Wiley-Blackwell; 2009:

12. Tsuta K, Ishii G, Nitadori J, Murata Y, Kodama T, Nagai K, Ochiai A: Comparison of the immunophenotypes of signet-ring cell carcinoma, solid adenocarcinoma with mucin production, and mucinous bronchioloalveolar carcinoma of the lung characterized by the presence of cytoplasmic mucin. J Pathol 2006, 209:78-87.

13. Matsukuma S, Yoshihara M, Kasai F, Kato A, Yoshida A, Akaike M, Kobayashi O, Nakayama H, Sakuma Y, Yoshida T, Kameda Y, Ysuchiya E, Miyagi Y: Rapid and simple detection of hot spot point mutations of epidermal growth factor receptor, BRAF, and NRAS in cancers using the loop-hybrid mobility shift assay. J Mol Diagn 2006, 8:504-512.

14. Walsh MM, Bleiweiss IJ: Invasive micropapillary carcinoma of the breast: eighty cases of an under-recognized entity. Hum Pathol 2001, 32:583-589.

15. Johansson SL, Borghede G, Holmang S: Micropapillary bladder carcinoma: a clinicopathological study of 20 cases. J Urol 1999, 161:1798-1802.

16. Kim M, Hong S, Jang S, Yu E, Kim J, Kim K, Gong G, Ro J: Invasive colorectal micropapillary carcinoma: an aggressive variant of adenocarcinoma. Hum Pathol 2006, 37:809-815.

17. Nora S, Presmanes MC, Monroy V, Alandro MH, Alvarez-Fernandez E: Micropapillary lung adenocarcinoma: a distinctive histologic subtype with prognostic significance. Cases series. Hum Pathol 2008, 39:324-330.

18. Kamiya K, Hayashi Y, Douguchi J, Hashiguchi A, Yamada T, Izumi Y, Watanabe M, Kawamura M, Horinouchi H, Shimada N, Kobayashi K, Sakamoto M: Histopathological features and prognostic significance of the micropapillary pattern in lung adenocarcinoma. Mod Pathol 2008, 21:992-1001.

19. Nieto MA: Epithelial-mesenchymal transitions in development and disease: old views and new perspectives. Int I Dev Biol 2009, 53:1541-1547.

20. Ponta H, Sherman L, Herrlich PA: CD44: from adhesion molecules to signaling regulators. Nat Rev Mol Cell Biol 2003, 4:33-45.

21. Elliot $B E$, Hung $W L$, Boag $A H$, Tuck $A B$ : The role of hepatocyte growth factor (scatter factor) in epithelial-mesenchymal transition and breast cancer. Can J Physiol Pharmacol 2002, 80:91-102.

22. Orian-Rousseau V, Chen L, Sleeman JP, Herrlich P, Ponta H: CD44 is required for two consecutive steps in HGF/c-Met signaling. Genes Dev 2002, 16:3074-3086.

23. Jiang F, Caraway NP, Bekele BN, Zhang HZ, Khanna A, Wang H, Li R, Fernandez RL, Zaidi TM, Johnston DA, Katz RL: Surfactant protein A gene deletion and prognostics for patients with stage I non-small cell lung cancer. Clin Cancer Res 2005, 11:5417-5424.

24. Tsutsumida H, Nomoto M, Goto M, Kitajima S, Kubota I, Hirotsu Y, Wakimoto J, Hollingsworth MA, Yonezawa S: A micropapillary pattern is predictive of a poor prognosis in lung adenocarcinoma, and reduced surfactant apoprotein A expression in the micropapillary pattern is an excellent indicator of a poor prognosis. Modern Pathol 2007, 20:638-647.

25. Ninomiya H, Hiramatsu M, Inamura K, Nomura K, Okui M, Miyoshi T, Okumura S, Satoh Y, Nakagawa K, Nishio M, Horai T, Miyata S, Tsuchiya E, Fukuyama E, Ishikawa Y: Correlation between morphology and EGFR mutations in lung adenocarcinomas Significance of the micropapillary pattern and the hobnail cell type. Lung Cancer 2009, 63:235-240.

26. Inamura $\mathrm{K}$, Ninomiya $\mathrm{H}$, Ishikawa $\mathrm{Y}$, Matsubara $\mathrm{O}$ : Is the epidermal growth factor receptor status in lung cancers reflected in clinicopathologic features? Arch Pathol Lab Med 2010, 134:66-72.

27. Motoi N, Szoke J, Riely GJ, Seshan VE, Kris MG, Rusch WW, Gerald WL, Travis WD: Lung adenocarcinoma: modification of the 2004 WHO mixed subtype to include the major histologic subtype suggests correlations between papillary and micropapillary adenocarcinoma subtypes, EGFR mutations and gene expression analysis. Am J Surg Pathol 2008, 32:810-827. 
28. Kim YH, Ishii G, Goto K, Nagai K, Tsuta K, Shiono S, Nitadori J, Kodama T, Nishiwaki Y, Ochiai A: Dominant papillary subtype is a significant predictor of the response to gefitinib in adenocarcinoma of the lung Clin Cancer Res 2004, 10:7311-7317.

doi:10.1186/1746-1596-7-3

Cite this article as: Ohe et al: Stromal micropapillary component as a novel unfavorable prognostic factor of lung adenocarcinoma. Diagnostic Pathology 2012 7:3.

Submit your next manuscript to BioMed Central and take full advantage of:

- Convenient online submission

- Thorough peer review

- No space constraints or color figure charges

- Immediate publication on acceptance

- Inclusion in PubMed, CAS, Scopus and Google Scholar

- Research which is freely available for redistribution

Submit your manuscript at www.biomedcentral.com/submit
() Biomed Central 